\section{P-33 DIGITAL BEREAVEMENT TRAINING TO PRODUCE COMPASSIONATE EMPLOYERS AND PUBLIC FACING ORGANISATIONS}

Laura Barrett, Felicity Dewhurst. St Oswald's Hospice, Newcastle upon Tyne, UK

10.1136/spcare-2021-Hospice.54

Background Marie Curie's recent 'Better End of Life Care' report highlighted a lack of bereavement support nationally despite excess need exacerbated by COVID-19. Bereavement support should increase peoples' ability to cope and improve wellbeing, fundamental to enable bereaved individuals to return to work. Systems approaches to mass bereavement must create compassionate employers.

Aims To produce and evaluate a digitally delivered bereavement training package for employers and public-facingorganisations.

Methods St Oswald's Hospice's regional bereavement service started providing bereavement training for public and private sector employers in 2021. This unique enterprise teaches individuals in positions which support staff wellbeing about the experience and impact of being bereaved, how they can support bereaved employees and why it is important. Participants are asked to express how confident they feel supporting someone who has been bereaved both before and after the training and to complete a quantitative and qualitative evaluation.

Results 229 participants from 59 nationwide organisations including councils, charities, universities, and health-services have been trained. Those that were confident to help and support someone who has been bereaved increased from 21\% before to $76 \%$ after training. Participants also felt more comfortable talking about death and dying (34\% before, $78 \%$ after training). $98 \%$ of participants felt their expectations of the training were met and $88 \%$ stated they would recommend the training. Qualitative comments focussed on the sessions being informative, practical, and well delivered.

'The session was very informative and was delivered with great sensitivity. I'd highly recommend this to anyone working in or leading a team.'

Conclusion This project demonstrates that bereavement training can be effectively delivered to a wide range of employers and public-facing-organisations, increasing participants' confidence in supporting employees who have been bereaved. The next phase of this project is to expand training and perform qualitative evaluation with employers, including assessing whether training has resulted in modifications to participating organisations' bereavement policies.

\section{P-34 INCORPORATING MINDFULNESS MEDITATION INTO A THERAPEUTIC BEREAVEMENT GROUP}

Laura Smith, Caroline Allen. Peace Hospice, Watford, UK

\subsection{6/spcare-2021-Hospice.55}

Background The role of hospices in providing bereavement support is well-established (Department of Health, 2008). One effective strategy is the provision of therapeutic bereavement groups (Spence \& Smale, 2015; Vlasto, 2009) where participants receive emotional comfort from being with others who have undergone similar experiences, with a professional facilitator. However, previous service users often report feeling emotionally drained at the end of each session, and for a short period afterwards. Therefore we decided to explore the impact of incorporating a self-care activity at the end of each session.

Aims To investigate the effectiveness of a mindfulness meditation activity at the end of each bereavement group session on the subsequent mood of participants. The benefits of mindfulness meditation include improved feelings of wellbeing (Carmody \& Baer, 2008) and reduced psychological distress (Coffey \& Hartman, 2008) and could therefore be expected to lift the mood of participants after each group session.

Methods Participants completed a Core 10 assessment tool at the beginning and end of a six-week bereavement group programme to measure any overall changes in mood and functioning. Qualitative feedback was sought in the form of a participant questionnaire with open questions. Participants also gave verbal feedback with a particular focus on the incorporation of the self-care activity.

Results A related $t$ test revealed a statistically significant difference between beginning and end Core 10 scores $p \leq 0.05$. Verbal feedback indicated that participants generally left each session in a positive mood, and a word cloud analysis of written feedback revealed that 'uplifted', 'calm' and 'relaxed' were most frequently chosen to describe the mindfulness meditation activity.

Conclusion Including a self-care activity at the end of a therapeutic bereavement group session is beneficial in improving the mood of participants and may mitigate against the emotionally draining experience of participating in such a group. Further research into the most beneficial self-care activity would be useful for the planning and delivery of future groups.

\section{P-35 ENGAGING HOSPICE INPATIENTS TO SHARE A CONVERSATION ABOUT 'WHAT'S MOST IMPORTANT TO $\mathrm{ME}^{\prime}$}

Karen Jackson, Louise McKay. Havens Hospices, Southend-on-Sea, UK

\subsection{6/spcare-2021-Hospice.56}

Introduction Engaging people who have life-limiting conditions to talk about what matters most to them should be a key activity of all palliative care services. 'What's most important to me?' is more than just clinical care, it is: What I really care about; What is special to me; What I want to do or achieve.

Aims Our aim is to give every individual that comes into our care the opportunity to share with us what is most important to them (if they wish to do so), enabling: Provision of holistic, person-centred care; the courage to dream and aspire to achieve in whatever time they have; support in opening those conversations with those close to them.

Methods

- Care Team Journal Club shared article and 'What Matters Conversations' film and thereafter were keen to progress this idea for improvement.

- A nurse shared her experience of using slate hearts to record people's wishes at another hospice.

- Quality improvement methodology applied to test the idea.

- Large blue heart poster created for individuals to write on as they wish. 